\title{
Sol-Gel mediated facile synthesis of Zinc-Oxide nanoaggregates, their characterization and antibacterial activity
}

\author{
Vinay Sharma \\ Centre for Converging Technologies, University of Rajasthan, Jaipur, Rajasthan
}

\begin{abstract}
Zinc oxide nanoparticles have been known for their strong antimicrobial properties since a long time. I have synthesized $\mathrm{ZnO}$ nanoaggregates using the environment friendly Sol-Gel method. The particles thus prepared were characterized by various technique like UV vis spectrophotometer, X-ray diffraction and Scanning Electron Microscopy. These techniques confirmed the presence of $\mathrm{ZnO}$ nanoparticles in form of aggregates. Finally, the antibacterial nature of $\mathrm{ZnO}$ nanoaggregates was performed on E.coli MTCC 40 strain of gram negative bacteria using Disk diffusion method.
\end{abstract}

Keywords - antibacterial nature, disk diffusion method, E.coli MTCC40, Sol-Gel method, nanoaggregates

\section{Introduction}

Nanotechnology is the science of production, manipulation and use of materials at subatomic level to produce novel products and processes[1]. At this particular range various optical, biological, magnetic, physical and chemical properties of particles get changed drastically. Nanotechnology has tremendous applications in diagnostic devices, drug delivery, tissue engineering, environmental chemistry, water filtration, producing ecofriendly energy production systems, quantum computers etc. Due to high surface to volume ratio, nanoparticles have emerged as novel antibacterial agents[2]. The considerable antimicrobial activities of various nanoparticles, such as silver nanoparticles, $\mathrm{ZnO}, \mathrm{MgO}, \mathrm{TiO} 2$ and $\mathrm{SiO} 2$, and their selective toxicity to biological systems suggests a potential application as therapeutics, diagnostics, surgical devices and nanomedicine-based antimicrobial agents[3]. In the present study $\mathrm{i}$ have tried to synthesize $\mathrm{ZnO}$ nanoaggregates by using an economic and eco-friendly Sol-Gel method. This method has been proved very useful for production of metal oxides[4]. After synthesis, the particles were characterized by UV- vis spectrophotometer, X-ray diffractometer and SEM. For antibacterial activity demonstration, E.coli MTCC 40 strain of gram negative bacteria was used and disk diffusion method[5] was employed for it. I have divided the study in three sections, Synthesis of $\mathrm{ZnO}$ nanoaggregates, Characterization and Antibacterial activity.

\subsection{Chemicals and apparatus.}

\section{Experimental}

The chemicals used for the synthesis were Zinc acetate $5.487 \mathrm{~g}$ in $50 \mathrm{ml}$ dd water, Oxalic acid $1.096 \mathrm{~g}$ in $50 \mathrm{ml} \mathrm{dd} \mathrm{water.} \mathrm{A} \mathrm{magnetic} \mathrm{stirrer} \mathrm{cum} \mathrm{heater} \mathrm{was} \mathrm{to} \mathrm{carry} \mathrm{out} \mathrm{hydrolysis} \mathrm{of} \mathrm{precursors.} \mathrm{An} \mathrm{electric} \mathrm{furnace}$ upto temperature tolerence of $1000^{\circ} \mathrm{C}$ was used. Double distilled deionised MQ water was used.

\subsection{Synthesis of $\mathrm{ZnO}$ nanoaggregates.}

Zinc acetate $5.487 \mathrm{~g}$ was weighed and mixed well in $50 \mathrm{ml}$ dd water with the help of stirrer for 1 hour. In another beaker oxalic acid $1.096 \mathrm{~g}$ was weighed and mixed well in dd water for $1 / 2$ hour. The oxalic acid solution was then mixed in Zinc acetate solution dropwise with continous stirring for 3 hours. The resultant white colored solution was then washed with dd water several times to free off the acetate ions. It was then centrifuged at 5000rpm for 20 minutes. The resulting white gel precipitate was then heated at $87^{\circ} \mathrm{C}$ for 5 hrs.Finally, it was put in pre-calibrated electric furnace at $600^{\circ} \mathrm{C}$ for $2 \mathrm{hrs} . \mathrm{ZnO}$ nanoparticles were thus obtained in white powder form. Fig. 1 shows the resultant gel after $87^{\circ} \mathrm{C}$ heating.

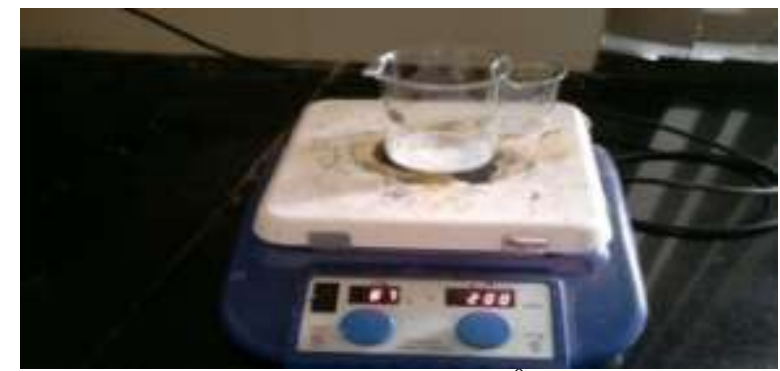

Fig.1 Heating at $87^{\circ} \mathrm{C}$ 


\subsection{Characterization.}

For characterization of above formed $\mathrm{ZnO}$ sample three different techniques were used i.e, SHIMAZDU UV-1800 uv vis spectrophotometer, X'PERT POWDER $\mathrm{x}$ ray diffractometer and Scanning electron microscope.

\subsection{Antibacterial activity.}

Prepared stock of gram negative bacteria E.coli MTCC40 was taken and its inoculum was streaked onto NA plate. By using 10x dilution and streaking, single colony of bacterium was picked up by sterilized inoculating loop.The whole work was performed in laminar flow hood. Pure line culture of E.coli MTCC40 was thus obtained. Briefly Nutrient Agar(NA) medium was used to cultivate bacteria.Fresh overnight cultures of inoculum $(100 \mu \mathrm{l})$ of E.coli bacteria was onto NA plates. Paper disks of $5 \mathrm{~mm}$ diameter were cut and autoclaved.Some of disks were dipped into standard vancomycin antibiotic $(10 \mu \mathrm{g} / \mathrm{litre})$, some into standard

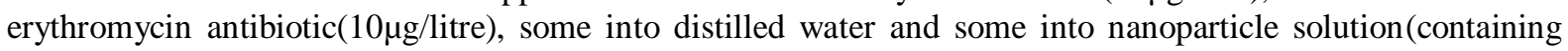
$50 \mathrm{mg} / \mathrm{litre} \mathrm{ZnO}$ nanoparticles) . These disks were placed on the surface of agar using flame sterilized forceps.

\subsection{Synthesis result}

\section{Results And Discussion}

After heating at $600^{\circ} \mathrm{C}$, the resultant is obtained in white powder form. It is shown in Fig.2.

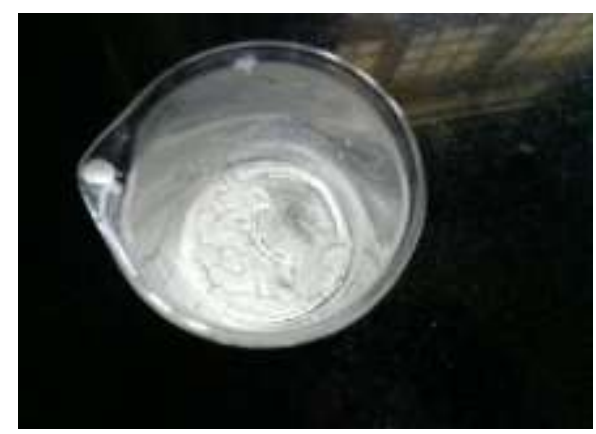

Fig.2. $\mathrm{ZnO}$ nanoaggregates

\subsection{Characterization results}

Fig.3. shows the UV vis absorption spectra of thus obtained $\mathrm{ZnO}$ nanoaggregates. A typical exciton peak at $368 \mathrm{~nm}$ is observed in the absorption spectrum. By comparing the result with standard UV absorption of bulk $\mathrm{ZnO}$ (at $380 \mathrm{~nm}$ ), it is evident that absorption peak shifts towards lower wavelength i.e. blue shift. This shift in the absorption edge is due to quantum size effect [6].

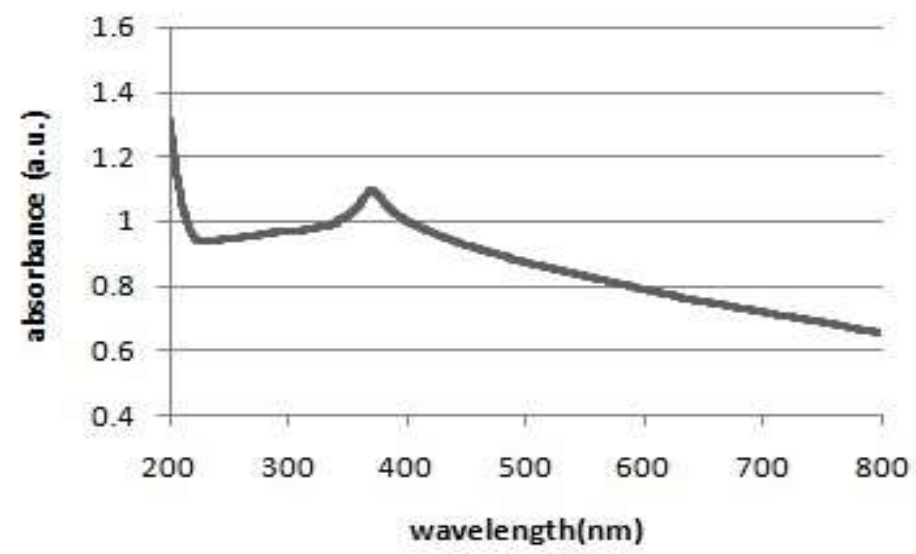

Fig.3. UV vis absorption spectrum of $\mathrm{ZnO}$ nanoaggregates.

Fig.4. shows the XRD pattern of $\mathrm{ZnO}$ nanoaggregates. The source of radiation was $\mathrm{CuK} \alpha$ with $\lambda=1.54 \mathrm{~A}^{0}$. All the diffraction data are in good aggreement with JCPDS files no.36-1451. However, in my results the XRD peaks are narrow showing that the particle size is more. This could be due to self aggregation of $\mathrm{ZnO}$ particles. The sharpness of peaks are showing that particles have good crystallinity. 


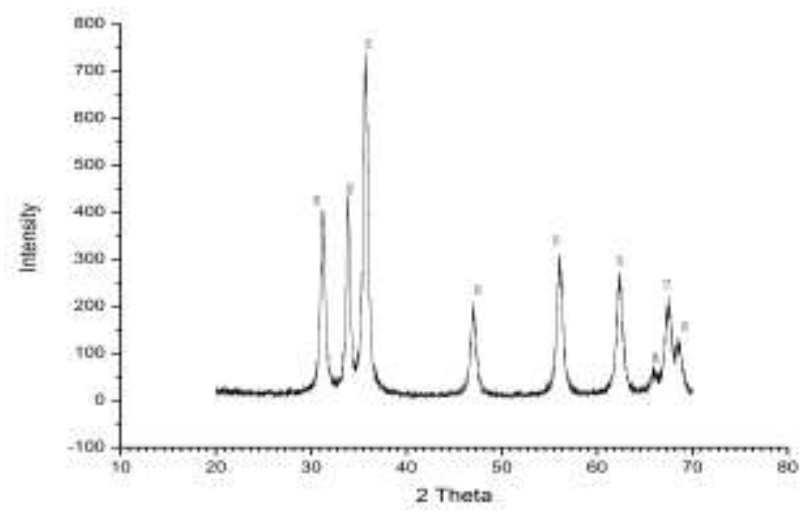

Fig.4 XRD pattern of $\mathrm{ZnO}$ nanoaggregates.

Fig. 5 shows the Scanning Electron Microscope (SEM) image of as prepared sample. This SEM image clearly shows that the particles have been aggregated with dimension of each aggregate being $\sim 2 \mu$ m.It thus justifies that $\mathrm{ZnO}$ nanoaggregates have been formed.

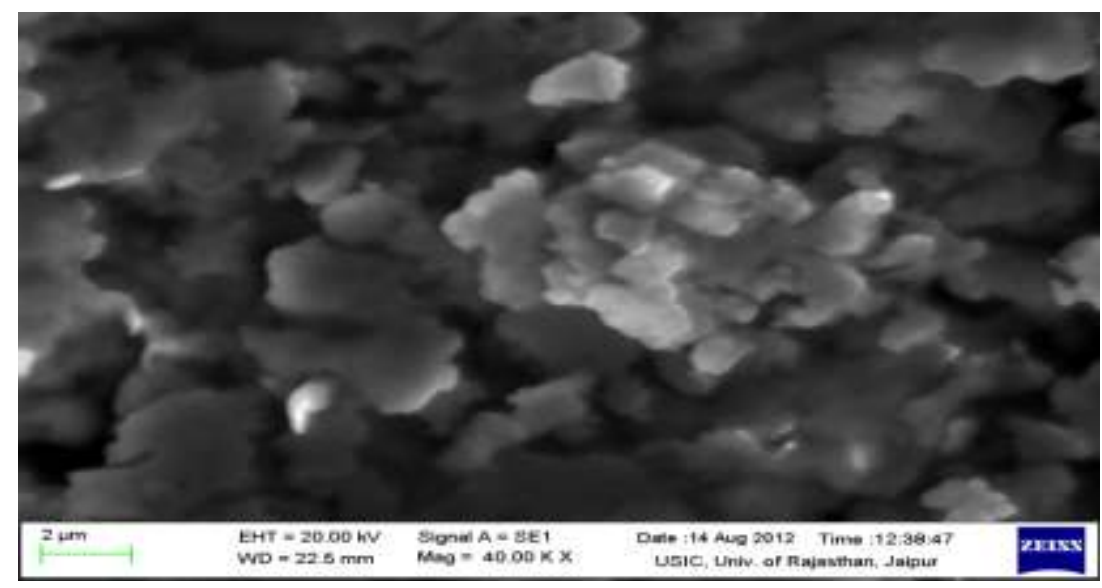

Fig.5 SEM image of $\mathrm{ZnO}$ nanoaggregates.

\subsection{Antibacterial activity results}

Fig.6 shows the antibacterial activity result of $\mathrm{ZnO}$ nanoaggregates. It is clearly visible that the zone of inhibition is highest for $\mathrm{ZnO}$ nanoaggregates indicated by(1). For erythromycin zone of inhibition indicated by(2), is lesser than $\mathrm{ZnO}$ nanoaggregates. (3) and (4) represents the activity of vancomycin and dd water respectively. Therefore, with gram negative E.coli MTCC40 strain, $\mathrm{ZnO}$ nanoaggregates were found to be the most effective, followed by erythromycin and vancomycin respectively.

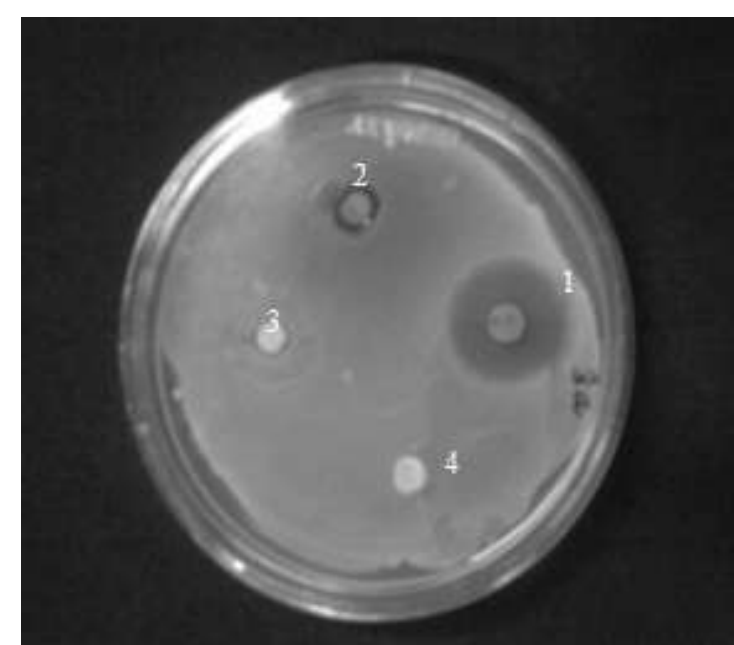

Fig.6 Antibacterial activity : (1) $\mathrm{ZnO}$ nanoaggregates > (2) Erytromycin > (3) Vancomycin > (4) dd Water 


\section{Conclusion}

In the present work i have reported the synthesis of $\mathrm{ZnO}$ nanoaggregates by a facile,eco-friendly and economic Sol-Gel method. UV vis absorption spectra clearly shows a blue shift which justifies the quantum confinement effect, hence lowering of dimensions. XRD pattern shows that the $\mathrm{ZnO}$ nanoaggregates have good crystallinity. SEM image clearly confirms the morphology of $\mathrm{ZnO}$ nanoaggregates. This aggregation could be due to high temperature $\left(600^{\circ} \mathrm{C}\right)$ heating.In the end of the study, antibacterial activity reveals that $\mathrm{ZnO}$ nanoaggregates are good at decreasing the bacterial growth.They are much effective than the traditionally used antibiotics.

\section{Acknowledgement}

I am thankful to Centre for Converging Technologies and Vigyan Bhawan, University of Rajasthan for their continuous support throughout the completion of this work.

\section{References}

[1] Alan L. Porter, Jan Youtie, Philip Shapira,David J. Schoeneck, Refining search terms for nanotechnology, J Nanopart Res (2008) 10:715-728,DOI 10.1007/s11051-007-9266-.

[2] Rizwan, W., Young-Soon, K., Amrita, M., Soon-Il, Y., Hyung-Shik, Sh., 2010. Forma-tion of ZnO-micro flowers prepared via solution process and their antibacterial activity. Journal of Nanoscale Research Letters 5, 1675-1681.

[3] M. Vijayakumara,, K. Priyab, F.T. Nancyb, A. Noorlidaha, A.B.A. Ahmeda , Biosynthesis, characterisation and anti-bacterial effect of plant-mediated silver nanoparticles using Artemisia nilagirica, Industrial Crops and Products (impact factor: 2.47). $01 / 2013 ; 41: 235-24$. .

[4] Sulabha k. kulkarni, Sol-gel method, in Synthesis of Nanomaterials-2(Chemical methods) Nanotechnology: Principles and Practices (New Delhi: Capital Publishing Company,2007) 93-94.

[5] Lin Jiang,Comparison of disk diffusion, agar dilution, and broth microdilution for antimicrobial susceptibility testing of five chitosans, A Thesis submitted to the Graduate Faculty of the Louisiana State University and Agricultural and Mechanical College in partial fulfillment of the requirements for the degree of Master of Science in The Department of Food Science, August 2011.

[6] Soosen Samuel M, Lekshmi Bose and George KC*, Optical properties of ZnO nanoparticles, Academic Review 2009: 57-65, ISSN: 0973-7464 Vol. XVI: No. $1 \& 2$ SB. 